\title{
Poursuivre le dialogue sur la politisation
}

Mounia Bennani-Chraïbi

\section{INTRODUCTION}

En 2010, un rapport du PNUD s'inquiète de «l'apathie» et du «manque d'engagement» manifestés par les 18-29 ans en Égypte ${ }^{1}$. L'année suivante, «la jeunesse » est érigée en icône des soulèvements qui agitent d'abord la Tunisie, puis l'Égypte et d'autres pays du Maghreb et du Moyen-Orient. À l'instar d'autres révolutions, les soulèvements de 2011 provoquent un séisme mental, relançant ainsi d'anciens débats épistémologiques ${ }^{2}$. À cette occasion, des chercheurs s'interrogent sur les œillères qui les auraient empêchés de percevoir les dynamiques à l'œuvre et appellent de leurs vœux les «échanges inter paradigmatiques ${ }^{3}$ ». Plus que jamais, il semble légitime de croiser les regards sur les résistances, les mouvements sociaux et les révolutions. L'une des gageures est de contourner les effets d'occultation produits par la division des tâches interne à un champ académique du reste hiérarchisé. Celle-ci dresse des barrières plus ou moins infranchissables entre des disciplines (science politique, sociologie, anthropologie, histoire, etc.), des domaines d'études (élections, partis politiques, politiques publiques, mouvements sociaux, révolutions, etc.), des contextes politiques (démocratique versus autoritaire) ou géoculturels («l'Occident» versus les « aires culturelles »), des langues et des lieux de production de savoir.

Dès les années 1980, Politique africaine figure parmi les pionniers de ces « appels d'air(e $)^{4} »$. La revue a posé des jalons importants pour explorer des « objets politiques non identifiés ${ }^{5}$ » et pour renouveler la réflexion sur les «modes d'action politique », tout en revisitant d'autres objets classiques de la science politique. Face à la richesse de ces travaux, cet article mobilise la notion de "politisation » pour suggérer des articulations entre des perspectives d'analyse concurrentes. Bien que le caractère fourre-tout de ce "concept éponge ${ }^{6}$ » et son usage « inflationniste » soient souvent relevés, celui-ci n'en garde pas moins de l'«intérêt pour désigner des processus très divers de construction d'un rapport au politique (...) dans des temps et à des échelles différents ${ }^{7}$ ». Dans un premier temps, nous présenterons quelques-unes des tendances qui ont animé les discussions à ce sujet et leur fécondation par les études aréales et en particulier africaines. Ensuite, nous poursuivrons le dialogue en invitant à penser ensemble - plutôt qu'à mettre en opposition - des politisations différentielles. En examinant des actions protestataires qui ont eu lieu au Maroc en 2018, nous essaierons de débusquer les intrications

\footnotetext{
${ }^{1}$ Des analyses fondées sur les données du Word Values Survey de 2008. United Nations Development Programme et Institute of National Planning, Egypt Human Development Report 2010. Youth in Egypt: Building our Future, UNDP - PNUD, 2010. Disponible sur : http://hdr.undp.org/en/content/2010-egypt-human-development-reportyouth-egypt, consulté le 11 mars 2021.

2 Sur ce débat, voir par exemple M. Bennani-Chraïbi, «Beyond Structure and Contingency: Toward an Interactionist and Sequential Approach to the 2011 Uprisings », Middle East Critique, vol. 24, no 4, 2017 , p. 373-395.

${ }^{3}$ M. Pace et F. Cavatorta, "The Arab Uprisings in Theoretical Perspective - An Introduction", Mediterranean Politics, vol. 17, $\mathrm{n}^{\circ} 2,2012$, p. 125-138.

${ }^{4}$ Sur ces apports, voir M. Bennani-Chraïbi et O. Fillieule, «Appel d'air(e)», dans Résistances et protestations dans les sociétés musulmanes, Paris, Presses de Sciences Po, 2003, p. 17-42.

5 D.-C. Martin, «À la quête des OPNI (Objets Politiques Non Identifiés). Comment traiter l'invention du politique?», Revue française de science politique, vol. 39, nº 6, 1989, p. 793-815.

${ }^{6}$ M. Aït-Aoudia, M. Bennani-Chraïbi, J.-G. Contamin, « Indicateurs et vecteurs de la politisation des individus : les vertus heuristiques du croisement des regards », Critique internationale, vol. 50, no 1, 2011, p. 9-20

${ }^{7}$ Y. Déloye, F. Haegel, «Chapitre 12 - Politisation: Temporalités et échelles» dans O. Fillieule, F. Haegel, C. Hamidi et V. Tiberj (eds.), Sociologie plurielle des comportements politiques: Je vote, tu contestes, elle cherche..., Paris, Presses de Sciences Po, 2017, p. 341.
} 
et les circulations entre des registres d'action pluriels, qui se déploient dans plusieurs sites d'interactions et qui impliquent un ensemble de participations et de médiations : «individuelles ou collectives, organisées ou informelles, dans ou en dehors de la légalité, à visée universaliste ou particulariste, autour de l'accès à des biens, à des droits, à la justice et à la reconnaissance ${ }^{8}$ ».

\section{I - LES FRONTIÈRES DU POLITIQUE, AU CAEUR DES DÉBATS SUR LA POLITISATION}

La politisation des individus et des groupes fait l'objet d'approches tantôt restrictives tantôt extensives, tout en donnant lieu à des tentatives de repérage. En filigrane s'esquisse la question des frontières : celles du politique ou de la politique, mais également celles qui sous-tendent le champ académique.

\section{I.1 Les approches restrictives du politique et de la politisation}

Dans les perspectives restrictives d'inspiration wébérienne, l'univers politique est associé à « un microcosme, c'est-à-dire un petit monde social relativement autonome à l'intérieur du grand monde social ${ }^{9}$ ». Pour Pierre Bourdieu, ce « champ de forces » est traversé par les luttes que se livrent des professionnels de la politique en concurrence pour la représentation du monde socia $^{10}$. De ce point de vue, la politique serait « une activité spécialisée régie par des règles qui définissent sa nature, ses conditions de prise en charge, ses modalités d'exercice : conçue comme champ spécifique, distinct des autres sphères d'activité sociales, la politique (politics) comporte un ensemble de rôles différenciés, qui font l'objet d'un strict encadrement ${ }^{11} \gg$.

Dans le prolongement de ces définitions étroitement articulées au processus occidental de civilisation électorale, la politisation des individus est associée au «mouvement ou [au] processus qui conduit [des individus, des catégories ou des groupes d'individus] à s'intéresser $\grave{a}$ et à s'impliquer dans la politique ${ }^{12}$. $\gg$ L'attention se fixe alors sur les rapports que les individus entretiennent avec le «champ politique», en tant que sphère différenciée, spécialisée et relativement autonomisée. Tandis que les perspectives socio-historiennes se centrent sur les processus d'acculturation électorale, les analyses inspirées par la sociologie bourdieusienne mettent l'accent sur la dépossession des moins dotés culturellement, économiquement et socialement ${ }^{13}$. Elles subordonnent "l'intérêt et l'attention accordés aux activités et aux productions du champ politique », au degré de « compétence politique » sur le plan cognitif (le fait d'être doté d'un savoir spécialisé permettant de différencier, de classer et de situer les acteurs) et statutaire (un sentiment de compétence ou d'indignité), qui varie en fonction du niveau d'instruction, et «à niveau d'instruction égal, avec le sexe, l'âge et, plus faiblement, le milieu social $»^{14}$.

Ces approches légitimistes présentent trois limites en particulier. D'une part, elles appréhendent la politisation sous le prisme de la démocratie représentative, avec une focale sur l'arène

\footnotetext{
${ }^{8}$ M. Bennani-Chraïbi, «Jeux de miroir de la "politisation": les acteurs associatifs de quartier à Casablanca», Critique internationale, vol. 1, $\mathrm{n}^{0}$ 50, 2011, p. 55-71.

${ }^{9}$ P. Bourdieu, Propos sur le champ politique, Lyon, Presses Universitaires Lyon, 2000, p. 52.

${ }^{10}$ P. Bourdieu, «La représentation politique [Éléments pour une théorie du champ politique] », Actes de la recherche en sciences sociales, vol. 36-37, mars 1981, p. 3-24.

${ }^{11}$ J. Chevallier, « Synthèse », dans CURAPP, La politique ailleurs, Paris, Presses Universitaires de France, 1998, p. 405 .

${ }^{12}$ Y. Déloye, F. Haegel, «Chapitre 12 - Politisation : Temporalités et échelles », art. cité, p. 325.

${ }_{13}$ Pour la restitution de ces débats dans une perspective historiographique, voir Y. Déloye, F. Haegel, "Chapitre 12 - Politisation: Temporalités et échelles», art. cité ; M. Offerlé, "Capacités politiques et politisations : faire voter et voter, XIXe-XXe siècles (1)», Genèses, vol. 2, n 67, 2007, p. 131-149; M. Offerlé, «Capacité politiques et politisations : faire voter et voter, XIXe-XXe siècles (2) », Genèses, vol. 68, n 3, 2007, p. 131-149.

${ }^{14}$ D. Gaxie, Le cens caché. Inégalités culturelles et ségrégation politique, Paris, Seuil, 1993 [1978], p. 31-32.
} 
électorale, et se révèlent donc peu adéquates pour l'étude du politique en contexte autoritaire. D'autre part, elles rejettent dans le domaine de l' «apathie politique » et de «l'incompétence» une myriade de discours et de pratiques. Enfin, elles contraindraient les chercheurs qui analysent d'autres configurations politiques à «ne saisir le politique qu'à travers les modalités "officielles" de sa délimitation» par les dominants ${ }^{15}$. Au détour de terrains extra-occidentaux, des conceptions plus larges du politique et de la politisation se sont développées en dialogue, notamment, avec les anthropologues et les historiens qui ont insisté sur l'ambivalence et la fluidité du politique.

\section{I.2 Les conceptions extensives du politique et de la politisation}

Au cœur du projet éditorial de Politique africaine, se trouve l'aspiration de questionner et de repenser «l'appareil conceptuel de la science politique» employé jusque-là pour étudier les terrains africains. En 1981, dans l'avant-propos du premier numéro, sont repoussées dos à dos les grilles de lecture étiologiques et normatives «d'inspiration missionnaire » consistant à «établir une pathologie politique africaine», et les approches d'inspiration marxiste imprégnées par « un certain prophétisme empreint de finalisme et, parfois, de simplisme $»^{16}$. Par ailleurs, et à contre-courant des thèses sur l'apathie des citoyens ordinaires en contexte autoritaire, la revue donne une forte impulsion aux entreprises qui explorent le «politique par le bas ${ }^{17}$ ». Au cours des années 1990, les investigations sur la pluralité des «avenues de la participation ${ }^{18}$ » se multiplient dans les études aréales, mettant en évidence les formes quotidiennes de résistance à l'autorité ${ }^{19}$, «la politique du faire ${ }^{20}$ », ou encore «les arts de la rue $^{21} \gg$. Entre révolte ouverte et soumission absolue, se dresse ainsi un riche arsenal de subversion tant sur le plan discursif (expression de la déférence, jeux de langage codés, échanges en coulisse ou en public) que celui des pratiques («mauvaise volonté », pratiques culturelles, détournement de ressources, actions de sabotage, migrations...). Peu à peu, le « politique autrement » et les « porosités » des « frontières du politique » sont scrutés y compris à partir de terrains européens ${ }^{22}$, avec une attention plus grande à « la production sociale de la politique ${ }^{23} \gg$.

Faut-il considérer comme politique toute tentative de griller les frontières ou toute dissimulation d'une partie de sa récolte pour ne pas s'acquitter de l'impôt? Pour certains comme James Scott, une stratégie de survie n'est pas antinomique avec un acte de résistance, peu importe l'existence ou l'absence d'une intention de nuire aux puissants ${ }^{24}$. En revanche, d'autres travaux mettent en garde contre les risques de dilution du politique dans le social. À cet égard, les problèmes de repérage se posent explicitement dans l'avant-propos du premier numéro de Politique

\footnotetext{
${ }^{15}$ M. Aït-Aoudia, M. Bennani-Chraïbi et J.-G. Contamin, « Contribution à une histoire sociale de la conception lagroyenne de la politisation », Critique internationale, vol. 48, n 3, 2010, p. 207-220.

${ }^{16}$ C. Coulon, J.-F. Bayart et Y.-A. Fauré, « Avant-Propos », Politique africaine, vol. 1, no 1, 1981, p. 2-4.

${ }^{17}$ Voir J. F. Bayart, «Le politique par le bas. Questions de méthode», Politique africaine, vol. 1, n ${ }^{\circ} 1,1981, \mathrm{p}$. 53-82, et l'ouvrage qui rassemble une série d'articles publiés entre 1981 et 1990 : J. F. Bayart, A. Mbembe, C. Toulabor, Le politique par le bas en Afrique noire. Contributions à une problématique de la démocratie, Paris, Karthala, 1992.

${ }^{18}$ D. Singerman, Avenues of Participation: Family, Politics and Networks in Urban Quarters of Cairo, Princeton, Princeton University Press, 1995.

${ }^{19}$ Voir les travaux de J. Scott (par exemple : Weapons of the Weak: Everyday Forms of Peasant Resistance, New Haven/London, Yale University Press), ou encore : D. Haynes and G. Prakash, Contesting Power: Resistance and Everyday Social Relations in South Asia, Berkeley and Los Angeles, University of California Press, 1992.

${ }^{20}$ C. Goirand, La politique des favelas, Paris, Karthala, 2000.

${ }^{21}$ P. Haenni, L'ordre des caïds. Conjurer la dissidence urbaine au Caire, Paris/Le Caire, Karthala/CEDEJ, 2005.

${ }^{22}$ Cette tendance est inaugurée dans : CURAPP, La politique ailleurs, Paris, Presses Universitaires de France, 1998.

${ }^{23}$ J. Lagroye, « Avant-propos », La politisation, Paris, Belin, 2003, p. 4.

${ }^{24}$ J. Scott, Weapons of the Weak, op. cit., p. 26.
} 
africaine : « La question centrale à laquelle on est aujourd'hui confronté si l'on cherche à mieux comprendre cette vie politique en Afrique noire, a trait au repérage de ses modalités et au champ social qu'elle occupe ${ }^{25} \gg$.

Dans le prolongement des réflexions publiées dans cette revue, Jean-François Bayart a fourni « une solution opératoire, susceptible d'analyser le passage au politique des groupes sociaux subordonnés ${ }^{26} »$. Son point de départ est « une théorie de l'action », dans le sens de la « théorie de la structuration» de Giddens, et «aux antipodes de tout déterminisme structurel $»^{27}$. Sa démarche interdisciplinaire s'enrichit des travaux de Michel Foucault, Michel de Certeau, Michel Vovelle, ou encore du linguiste Mikhaïl Bakhtine. Elle vise à compenser le « retard pris par la science politique », et à réintroduire « le problème du pouvoir et de l'État» qui serait délaissé par ces travaux. Pour sa part, Bayart invite à repérer le "politique » sur la base de catégorisations à la fois savantes et émiques en soulignant l'importance du « regard du pouvoir qui confère la propriété du politique, soit qu'il la dénie à la pratique d'un acteur qui s'en réclame pourtant, soit qu'il l'attribue à un acteur qui s'en défend ou n'en peut mais - et dans les deux cas il agit généralement pour lui de mieux contrôler, de mieux réprimer $»^{28}$. À partir de là, il formule «une problématique des pratiques énonciatives des structures et des représentations politiques », qui s'accommode de l'indétermination, de l'inachèvement et de la polyphonie du politique $^{29}$.

Outre l'attention portée aux pratiques énonciatives, deux dimensions sous-tendront les modalités de repérage du politique proposées par des sociologues et des politistes qui travaillent sur des contextes démocratiques en s'inscrivant dans d'autres filiations intellectuelles que celles des auteurs de Politique africaine ${ }^{30}$. La première a trait à la conflictualité, et à son corollaire la régulation. Dès 1973, le politiste Jean Leca proclame que le politique est irréductible à un lieu, à des représentations, à des pratiques ou à des institutions : « tout est potentiellement politique, c'est le rapport de forces entre les groupes qui en détermine les frontières ${ }^{31} »$. Ce phénomène se combine souvent avec un deuxième processus : le passage du singulier au collectif qui se traduirait par une identification à un «nous », par une montée en généralité ${ }^{32}$, ou encore par l'énonciation de discours animés par «l'esprit public ${ }^{33} »$. D'une certaine manière, ces deux processus se retrouvent aussi dans le «"minimum commun dénominateur" philosophique de la subjectivation politique» qu'identifie Federico Tarragoni : «1) la subjectivation politique présuppose une dialectique entre sujet et commun, individuel et collectif. (...). 2) [elle] actualise un conflit qui, en suivant le critère précédent, fissure le sujet et repense le contrat démocratique $^{34} »$. Ce sociologue en déduit que les sciences sociales «étudient parfois les processus de subjectivation politique sans le savoir », dès lors qu' « elles s'intéressent également

\footnotetext{
${ }^{25}$ C. Coulon, J.-F. Bayart et Y.-A. Fauré, «Avant-Propos », art. cité, p. 3.

${ }^{26}$ J.-F. Bayart, «L'énonciation du politique », Revue française de science politique, vol. 3, no 35, 1985, p. 353354.

${ }^{27}$ Ibid., p. 354.

${ }^{28}$ Ibid., p. 367.

${ }^{29}$ Ibid., p. 358.

${ }^{30}$ Pour un état de la question, voir par exemple M. Aït-Aoudia et al., «Indicateurs et vecteurs de la politisation des individus... », art. cité.

${ }^{31}$ J. Leca, « Le repérage du politique », Projet, vol. 71, 1973, p. 12 et 16.

32 Voir par exemple W. Gamson, Talking politics, Cambridge, Cambridge University Press, 1992 ; C. Hamidi, «Éléments pour une approche interactionniste de la politisation. Engagement associatif et rapport au politique dans les associations locales issues de l'immigration », Revue française de science politique, vol. 56, $\mathrm{n}^{\circ}$ 1, 2006, p. 5-25.

${ }^{3}$ N. Eliasoph, Avoiding Politics: How Americans Produce Apathy in Everyday Life, Cambridge, Cambridge University Press, 1998.

${ }^{34}$ F. Tarragoni, « Du rapport de la subjectivation politique au monde social. Les raisons d'une mésentente entre sociologie et philosophie politique », Raisons politiques, vol. 2, nº 62, 2016, p. 115-130.
} 


\section{Preprint mars 2021}

aux modalités par lesquelles une action politique engage une refonte de l'identité individuelle et des collectifs politiques d'appartenance ».

\subsection{Vers la remise en cause de la division des tâches interne au champ académique}

Le fait de questionner ou de revisiter les frontières du politique et de la participation politique a autorisé le décloisonnement entre plusieurs domaines d'études, favorisant ainsi la «banalisation » de l'exotisme à laquelle appelaient de leurs vœux Michel Camau ou Christian Coulon au milieu des années $1990^{35}$. Ici, nous évoquerons deux axes principaux : les propositions d'articulation entre résistances, mouvements sociaux et révolutions; le regain d'intérêt pour des « objets délaissés » ayant trait à la politique instituée.

Croiser les regards par-delà la division des tâches interne au champ académique entraîne, en premier lieu, la remise en cause du «lien consubstantiel postulé entre démocratisation et mouvement social et la distinction de nature, posée a priori, entre les répertoires d'action démocratiques (regroupant toute la palette des actions conventionnelles et non conventionnelles) et l'émeute, la révolte et l'agitation révolutionnaire ${ }^{36} »$. Ce déplacement crée les conditions de possibilité d'une discussion féconde avec les théories des mouvements sociaux, à partir de différents contextes géoculturels et politiques ${ }^{37}$. Par ailleurs, il ouvre la voie des conceptions de la participation politique sous forme d'un "continuum» entre actions conventionnelles et non conventionnelles, individuelles et collectives, qui sous-tend plusieurs travaux publiés dans Politique africaine ${ }^{38}$. Reste à souligner que ces perspectives ont des filiations et des ramifications plurielles.

Pionnières sont les recherches d'Asef Bayat sur « les mouvements de pauvres » en Iran, à la fin des années 1970 et au début des années 1980. Ce sociologue démontre que les « dominés » font de l'« empiétement silencieux du quotidien" (quiet encroachment of the ordinary) : ils ne se cantonnent pas aux tactiques de résistance solitaires et isolées; lorsque l'étau de la répression se desserre, ils recourent à des actions collectives organisées ${ }^{39}$. Pour sa part, l'anthropologue Partha Chatterjee observe des «enchevêtrements» dans les pratiques de squatters au sud de Calcutta $^{40}$. Cette figure des études postcoloniales appréhende les relations entre «gouvernés» et «gouvernants» dans le cadre de la «société politique»: un «terrain contingent» où les luttes puisent aussi bien dans le registre du droit que dans la " para-légalité », laissant entrevoir une diversité d'instances de médiation ${ }^{41}$.

\footnotetext{
${ }^{35}$ Camau Michel, «Une science politique entre deux ordres de justification », rapport introductif au cinquième congrès de l'Association française de science politique, table ronde ${ }^{\circ} 1,1996$; C. Coulon, «L'exotisme peut-il être banal ?», Politique africaine, ${ }^{\circ} 65,1997$, p. 77-95.

${ }^{36}$ M. Bennani-Chraïbi et O. Fillieule, « Appel d'air(e)», art. cité., p. 9.

${ }^{37}$ Parmi les pionniers : E. I. Burke et I. Lapidus, Islam, Politics and Social Movements, Berkeley, University of California Press, 1988. Pour un état de la question stimulant sur les usages de la sociologie des mobilisations en Afrique, voir J. Siméant, «Protester/mobiliser/ne pas consentir. Sur quelques avatars de la sociologie des mobilisations appliquée au continent africain », Revue internationale de politique comparée, vol. 20, $\mathrm{n}^{\circ} 2,2013$, p. $125-143$.

${ }^{38}$ Parmi les travaux publiés avant 2011, voir notamment J. Lafargue, « La rue africaine en mouvement : politique et action collective », Politique Africaine, $\mathrm{n}^{\circ}$ 63, 1996, p. 24-38; M.-E. Pommerolle, «Routines autoritaires et innovations militantes. Le cas d'un mouvement étudiant au Cameroun », Politique africaine, 4, n 108, 2007, p. 155-172; V. Veguilla, «Conflits et actions collectives autour de l'exploitation du poulpe au Maroc», Politique africaine, 4, n 116, 2009, p. 43-62; A. Allal, « Réformes néolibérales, clientélismes et protestations en situation autoritaire. Les mouvements contestataires dans le bassin minier de Gafsa en Tunisie (2008) », Politique Africaine, 1, no 117, 2010, p. 107-125; M. Catusse et F. Vairel, «Question sociale et développement: les territoires de l'action publique et de la contestation au Maroc », Politique africaine, 4, n 120, 2010, p. 5-23.

${ }^{39}$ A. Bayat, Street Politics. Poor People Movements in Iran, Columbia University Press, New York, 1997.

${ }^{40}$ P. Chatterjee, The Politics of the Governed. Reflections on Popular Politics in Most of the World, New York, Columbia University Press, 2004.

${ }^{41}$ Traduction dans P. Chatterjee, «Politique des gouvernés », Multitudes, vol. 2, nº 45, 16 mai 2011, p. $174-182$.
} 
Soucieux de faire dialoguer plusieurs traditions académiques, nous avons procédé au début des années 2000 à une mise en ordre en formulant la question suivante : "selon quelles logiques, en fonction des contextes et de leurs transformations, les individus ou les groupes se tournent vers des formes d'opposition actives débouchant plus ou moins sur la confrontation, soit au contraire se cantonnent à la débrouillardise, à la résistance passive, voire se résignent au silence, à la dissolution, à l'exil intérieur ou à l'émigration ${ }^{42} »$ ? Le fait même de rompre avec une conception des régimes politiques ${ }^{43}$, essentialisée et indexée sur un étalon de «la modernité » politique ${ }^{44}$, a des implications. Il conduit à examiner les (re)structurations de la conflictualité politique et des modalités de régulation, en prêtant attention à ce qui contraint et affecte les relations entre gouvernants et gouvernés, selon des temporalités plus ou moins longues et au croisement des « dynamiques du dedans » et des « dynamiques du dehors ${ }^{45}$.

Dans le sillage des soulèvements de 2011 s'esquissent de nouvelles propositions d'articulation entre théorie des mouvements sociaux, sociologie des révolutions, sociologie historique de l'État et régimes politiques comparés ${ }^{46}$. Elles permettent de penser à nouveaux frais les mobilisations qui se déploient dans différentes arènes du politique et leurs économies morales ${ }^{47}$, avec un intérêt accentué pour les évènements transformateurs qu'ils soient révolutionnaires ou contre-révolutionnaires ${ }^{48}$. La focale porte, à l'échelle des organisations et des répertoires d'action, sur les interactions et les circulations et, à l'échelle des individus, sur les processus d'engagement, de conversion, de désengagement, ou de radicalisation ${ }^{49}$.

Selon d'autres cheminements, les réflexions sur le jeu politique «institué » ou « officiel» connaissent un nouvel élan, en se démarquant des approches centrées sur les «dysfonctionnements» au regard d'un horizon d'attente démocratique ; elles secouent de la sorte « le carcan normatif qui empêche d'étudier "ce qui ne marche pas" 50 ». La posture se veut à nouveau « œcuménique ${ }^{51}$ », et il n'est pas question de prendre pour point de départ un «modèle». À partir de la fin des années 1990, les élections sont ainsi constituées en «objet scientifique » tout à fait « pertinent » en Afrique, surtout pour le nord de ce continent ${ }^{52}$, et des

\footnotetext{
${ }^{42}$ O. Fillieule et M. Bennani-Chraïbi, «Exit, voice, loyalty et bien d'autres choses...», dans Résistances et protestations dans les sociétés musulmanes, op. cit., p. 43-126, p. 68.

${ }^{43}$ Pour une lecture relativisant les approches binaires et dichotomiques des régimes politiques, voir M. Camau, G. Massardier (dir.), Démocraties et autoritarismes. Fragmentation et hybridation des régimes, Paris, Karthala, 2009.

${ }^{44}$ Pour rappel, elle est associée au processus de différenciation qui sous-tend la formation de l'État en Occident et qui se traduit notamment par une autonomisation relative de la sphère politique et par le passage à une forme de domination rationnelle-légale.

${ }^{45}$ Selon la terminologie de G. Balandier, Sens et puissance : les dynamiques sociales, Paris, Presses Universitaires de France, 1971.

${ }^{46}$ Voir par exemple le numéro spécial « Retour sur les situations révolutionnaires arabes », Revue française de science politique, vol. 62, $\mathrm{n}^{\circ} 5,2012$; A. Allal et T. Pierret, Au cour des révoltes arabes: Devenir révolutionnaires, Paris, Colin, 2013 ; J. Beinin et F. Vairel, Social Movements, Mobilization, and Contestation in the Middle East and North Africa, 2d Edition, Stanford, Stanford University Press, 2013.

47 Voir par exemple B. Hibou, «Tunisie. Économie politique et morale d'un mouvement social», Politique africaine, vol. 1, n ${ }^{\circ}$ 121, mars 2011, p. 5-22.

${ }^{48}$ Voir en particulier trois dossiers de Politique africaine : «La Tunisie en révolution », 1, n $\mathrm{n}^{\circ}$ 121, mars 2011 ; «Parlements de la rue. Espaces publics de la parole et citoyenneté en Afrique », 3, n 127, 2012 ; « Restaurations autoritaires », 2, $\mathrm{n}^{\circ} 146,12$ octobre 2017.

49 Voir à ce sujet le dossier : «La "radicalisation" aide-t-elle à mieux penser ? », Politique africaine, 1, n 149 , 2018.

${ }^{50}$ M. Gazibo, «Pour une réhabilitation de l'analyse des partis en Afrique », Politique africaine, 4, n ${ }^{\circ} 104,2006$, p. $5-17$.

51 J. Blondel, «Plaidoyer pour une conception œcuménique de l'analyse comparée », Revue internationale de politique comparée, vol. 1, nº 1, 1994, p. 5-18, cité par M. Gazibo, ibid.

${ }^{52}$ Clin d'œil au dossier «Des élections comme les autres» : R. Otayek, «Les élections en Afrique sont-elles un objet scientifique pertinent?», Politique africaine, $n^{\circ} 69,1998$, p. 3-11. L'intitulé de ce numéro fait lui-même
} 
publications de Politique africaine enrichissent la réflexion sur les angles morts de la sociologie électorale. Sur les plans épistémologique et méthodologique, se confirme l'intuition qu'il est nécessaire d'étudier les élections qui se déroulent en Afrique tout autant que « les autres », sans se cantonner au rôle de l'observateur qui inventorie les anomalies ou à celui du chroniqueur focalisé sur le détail des résultats électoraux. Le vote est conçu en tant que «fait social et matériel » et les élections dans ce qu'elles produisent "par-delà la légitimation démocratique, voire la relégitimation autoritaire, ou encore le (non)renouvellement des élites ${ }^{53}$ ». Plutôt que d'appréhender la fabrique de l'électeur et du citoyen dans un processus de différenciation et d'autonomisation de la sphère politique, les analyses portent sur les phénomènes d'encastrement du politique dans le social, et de «production matérielle de l'État et de l'électeur ${ }^{54} »$. Dans l'ensemble, ces travaux contribuent à revitaliser la réflexion sur le clientélisme politique ${ }^{55}$, sur le vote communautaire et sur la fraude électorale, désormais conçus comme des formes de participation politique à part entière, qui s'inscrivent « dans un continuum d'échanges sociaux ${ }^{56} \gg$. En ce sens, ils font également écho aux approches sociohistoriques, notamment sur le terrain hexagonale, selon lesquelles « la politisation n'est pas réductible à la diffusion ou à l'ingestion de catégories universelles venues d'en haut, mais doit être référée à une série de malentendus par lesquels des individus dotés de représentations différentes et contradictoires s'approprient par des usages multiples (subversion, dérision, contournement, enrôlement) les cadres dans lesquels ils "doivent" opérer ${ }^{57}$ ".

Néanmoins, l'étude des partis politiques en Afrique ne rencontre pas le même engouement que celle des élections ou des transitions démocratiques ${ }^{58}$. En 2006, une livraison de Politique africaine invite à revisiter cet « objet délaissé » ${ }^{59}$. Dans les années qui suivent, quelques travaux publiés par la revue trahissent une relative «réhabilitation de l'analyse des partis en Afrique ${ }^{60} »$, principalement sous l'angle des mobilisations partisanes. À titre d'exemple, Valeria Alfieri examine les usages différenciés de la question ethnique par le Palipehutu-FNL au Burundi depuis sa création dans la clandestinité en 1980 à sa légalisation en $2009^{61}$. Pour sa part, Lucie Revilla se penche sur l'ancrage social et les modes organisationnels du National Congress Party dans les quartiers populaires du Grand Khartoum, en prêtant une attention particulière aux « trajectoires de distinction » que l'organisation islamiste contribue à façonner

écho à l'ouvrage fondateur de G. Hermet, J. Linz, A. Rouquié (Des élections pas comme les autres, Paris, Presses de la Fondation nationale des sciences politiques, 1978).

53 S. Perrot, M.-E. Pommerolle et J. Willis Justin, «La fabrique du vote : placer la matérialité au cœur de l'analyse », Politique africaine, « Matérialités du vote », vol. 4, n 144, 2016, p. 5-26.

${ }^{54}$ Idem.

${ }^{55}$ Parmi les travaux qui ont accompagné ce tournant, voir J. Auyero, Poor People's Politics. Peronist Survival Networks \& the Legacy of Evita, Duke University Press, Durham \& London, 2000.

${ }^{56}$ R. Banégas, "Marchandisation du vote, citoyenneté et consolidation démocratique au Bénin », Politique africaine, "Des élections "comme les autres" », n 69, 1998, p. 75-87. Voir aussi dans la même revue L. Zaki, «Séduction électorale au bidonville : jouer de l'opulence, de la jeunesse ou du handicap Casablanca », Politique africaine, vol. 3, no 107, 2007, p. 42-61 ; M. Vannetzel, «"Ils nous ont déjà essayés !". Clientélisme et mobilisation électorale frériste en Égypte », Politique africaine, vol. 108, n 4, 2007, p. 47-66.

${ }^{57}$ M. Offerlé, « Capacité politiques et politisations : faire voter et voter, XIXe-XXe siècles (2) », Genèses, vol. 68, $\mathrm{n}^{\mathrm{o}} 3,2007$, p. 157.

${ }^{58}$ Il en va de même pour les syndicats, du moins avant le lancement de l'ANR «Syndicalisme au quotidien en Afrique », [https://anr.fr/Projet-ANR-18-CE26-0008], coordonnée par Alexis Roy.

${ }^{59}$ Dossier « Partis politiques d'Afrique. Retours sur un objet délaissé », Politique africaine, 4, n $104,2006$.

${ }^{60}$ M. Gazibo, « Pour une réhabilitation de l'analyse des partis en Afrique », art. cité. Dans la même veine, voir par exemple M. Catusse et K. Karam, Returning to Political Parties? Political Party Development in the Arab Word, Beirut, Lebanese Center for Policy Studies, 2010.

${ }^{61}$ V. Alfieri, «Le Palipehutu-FNL au Burundi. Dynamiques d'ethnicisation et de "désethnicisation" », Politique africaine, vol. 1, n 141, 2016, p. 169-190. 
à l'échelle locale ${ }^{62}$. À leur tour, ces enquêtes restituent dans toute leur épaisseur les modalités plurielles de production sociale du politique, dans ce qu'elles ont de structuré et de structurant, et dans les innovations politiques auxquelles elles frayent la voie. Mais, dans l'ensemble, les partis politiques suscitent plus d'intérêt au Maghreb et en Égypte que dans le reste du continent $^{63}$, un phénomène qui mériterait d'être analysé.

$\mathrm{Au}$ terme de cette rétrospective, nous espérons avoir montré à quel point les analyses du politique et de la politisation qui trouvent écho dans la revue Politique africaine réservent la part du lion aux ambivalences et aux hybridations. Bien davantage, leur richesse provient de leur propension à déjouer les frontières entre disciplines, entre contextes politiques et aires géoculturelles. Ce faisant, il devient possible de saisir dans un même mouvement un éventail de pratiques et de lieux du politique, autrement dit de penser ensemble plusieurs formes de politisation; ce que nous allons illustrer à partir de notre terrain marocain dans ce qui suit.

\section{II - PENSER ENSEMBLE PLUSIEURS FORMES DE POLITISATION}

Pour Albert O. Hirschman, la défection (exit), la prise de parole (voice) et la loyauté (loyalty) constituent les trois conduites possibles en cas de désaccord ou d'insatisfaction ${ }^{64}$. Au début des années 2000, l'une des réappropriations de ce célèbre triptyque visait à enrichir la panoplie des stratégies envisageables pour un acteur insatisfait ou en désaccord ${ }^{65}$. Tout en esquissant des formes de défection allant de l'exil intérieur à l'exil politique qui peut ouvrir la voie à une dissidence active, l'accent est mis sur les modalités de prise de parole. À un premier niveau, il est possible de protester d'une part de manière ouverte en restant dans le cadre légal ou en le quittant, ou d'autre part de manière larvée en développant des stratégies parallèles ou en prenant le pouvoir pour cible. À un deuxième niveau, il est envisageable de rester sans remettre en cause le pouvoir, soit en participant activement à la relation (loyauté), soit en s'accommodant ou en s'enfermant dans l'apathie ${ }^{66}$. À partir de là, en fonction de la légitimité accordée ou déniée au pouvoir, les acteurs optent pour le non-affrontement, l'affrontement ouvert, ou l'affrontement larvé. Une telle approche permet d'intégrer le fait que les stratégies sont contraintes par les répertoires d'action disponibles tant à l'échelle nationale que transnationale, de restituer la transformation des trajectoires au gré du niveau de répression exercé, de saisir les mouvements d'engagement et de désengagement dans toute leur épaisseur, sans négliger les phases de « mise en sommei ${ }^{67} \gg$.

Dans le prolongement d'un terrain de longue durée à Casablanca, nous suggérons de revisiter ces propositions sous l'éclairage des débats sur la politisation. Nous évoquerons en particulier un épisode observé à distance, et durant lequel des acteurs brandissent la menace de "l'exit» dans le cadre d'une action collective protestataire. Bien davantage, cette articulation entre «l'exit» et la «voice» laisse entrevoir une imbrication entre différents types de politisation saisie sous d'autres formes pendant des campagnes électorales ${ }^{68}$ : une sophistication politique qui se traduit par la maîtrise d'un ensemble de codes et de pratiques politiques, une politisation

\footnotetext{
${ }^{62}$ L. Revilla, «Les kayzan au quartier : milieu partisan et trajectoires de distinction dans les quartiers populaires du Grand Khartoum », Politique africaine, vol. 2, n 158, 2020, p. 33-55.

63 Voir par exemple M. Aït-Aoudia, L'expérience démocratique en Algérie (1988-1992). Apprentissages politiques et changement de régime, Paris, Presses de Sciences Po, 2015 ; M. Vannetzel, Les Frères musulmans égyptiens. Enquête sur un secret public, Paris, Karthala, 2016.

${ }^{64}$ A. O. Hirschman, Défection et Prise de parole. Théorie et applications, Paris, Fayard, 1995.

${ }^{65}$ O. Fillieule et M. Bennani-Chraïbi, «Exit, voice, loyalty et bien d'autres choses... », art. cité.

${ }^{66}$ En référence à G. Bajoit, « Exit, voice, loyalty... and apathy. Les réactions individuelles au mécontentement », Revue française de sociologie, XXIX, 1988, p. 325-345.

67 V. Taylor, «La continuité des mouvements sociaux. La mise en veille du mouvement des femmes », dans O. Fillieule (dir.), Le désengagement militant, Paris, Belin, 2005, p. 229-250.

68 M. Bennani-Chraïbi, «Politisations différentielles et acculturations mutuelles en contexte autoritaire: Ethnographie d'une inversion du cens électoral », Politix, vol. 113, nº 1, 2016, p. 141-169.
} 


\section{Preprint mars 2021}

par désingularisation ou par montée en généralité, ou encore le registre de la politique par le bas.

À la fin du mois de septembre 2018, près de 4000 habitants d'un douar ${ }^{69}$ de Casablanca proclament symboliquement leur volonté de se diriger vers Ceuta, l'enclave espagnole, et de demander l'asile humanitaire (al-luju' al-insani), pour protester contre la démolition de leurs logements. L'une de leurs actions protestataires est filmée et diffusée par un YouTubeur ${ }^{70}$. Situé dans la zone industrielle et maritime de Ain Sebaa à Casablanca, le douar Hsibou qui compte près de mille ménages est considéré comme un habitat insalubre ${ }^{71}$. Pour le Conseil de la ville de Casablanca, l'opération de démolition et de relogement s'inscrit dans le cadre du « combat» contre «la précarité à travers la lutte contre l'habitat insalubre», et deux options ont été proposées aux habitants : bénéficier d'un logement social pour le prix de 100000 dirhams, ou d'un lot à 22000 dirhams, pour construire un logement en binôme à Sidi Hajjaj, une zone rurale, située à $25 \mathrm{~km}$ de Douar Hsibou, et limitrophe de la plus grande décharge de la métropole. Quant aux habitants du douar, ils ont présenté un «dossier revendicatif » (mileff matlabi) qui préconise la réhabilitation de leur habitat à Ain Sebaa même. Outre le coût prohibitif du relogement, ils développent plusieurs arguments. Leur «exil»(tahjir) à Sidi Hajjaj les éloignera de leur lieu de subsistance, la mer et les usines d'Ain Sebaa. Il n'existe pas de moyens de transport adéquats entre les deux sites. Le lieu d'accueil ne dispose pas d'infrastructures de base (eau, électricité, écoles, dispensaires, etc.) et « le dossier du logement n'a pas été pensé en lien avec le dossier de l'emploi et le dossier de l'enseignement». Dès lors, en prétendant combattre la précarité et le chômage, les autorités ne feront que les amplifier. À cet égard, l'un des résidents reproche aux décideurs de lutter contre le logement dit non réglementaire ( 'achwa'i), en recourant à des procédés « irréguliers 》 ('achwa'iyya) et illégaux, rappelant que le tribunal s'est déclaré non compétent et n'a pas émis d'avis d'éviction ${ }^{72}$. Dans l'épisode filmé et diffusé, deux dimensions méritent une attention particulière : d'une part, les justifications qui articulent les sacrifices du douar pour la patrie, la renonciation à la nationalité et la demande de l'asile humanitaire ; d'autre part, les pièces du répertoire protestataire mobilisé.

«Le douar demande l'asile humanitaire », «le peuple veut la renonciation à la nationalité » (ach-cha'b yourid isqat al-jansiyya), etc. Au-delà de ces slogans, les témoignages mettent l'accent sur les "qualités» des habitants du douar: l'absence de criminalité, les « compétences » qu'il abrite, mais surtout son passé dans la lutte pour l'indépendance. L'un des intervenants explique que le douar « a une histoire », qu'il regroupe « des fils et des veuves de résistants ». Un autre présente devant la caméra une carte de résistant. À travers ces récits, la visite au lendemain de l'indépendance du roi Mohammed $\mathrm{V}$ au douar, attestée par une photo, est constituée en pièce centrale de la mémoire collective locale. À l'aune de cet épisode, des résidents de Sidi Hsibou dénoncent la menace qui pèse sur eux : «Aujourd'hui, on nous détruit nos logements, nous ne comptons plus dans ce pays, nous n'avons plus rien à y faire. Nous avons décidé de renoncer à notre nationalité. »

Sur un autre plan, l'acculturation au registre du «droit» est plus manifeste que jamais. Les protestataires présentent leur mobilisation comme « pacifique » et l'inscrivent dans «le cadre

\footnotetext{
${ }^{69}$ Dans ce contexte, il s'agit d'une zone d'habitat considérée comme non réglementaire.

${ }^{70}$ Le 30 septembre 2018, « Moul chekkara », un YouTubeur suivi par 872000 personnes sur Facebook, filme en direct des habitants du Douar Hsibou, qui protestent contre l'imminence de la destruction de leurs logis. Vidéo disponible sur: https://www.facebook.com/moul.chekara.officiel/videos/102914987322529/ (411 815 vues au 11 octobre 2018 ).

${ }^{71}$ Sur ces opérations de délogement, voir L. Hallaoui, « Ce que l'on sait de l'opération de délogement de douar El Ouasti à Casablanca », Al Huffington Post Maghreb-Maroc, 25 septembre 2018; L. Hallaoui, « Douar Hsibou : Après la destruction du bidonville, les opérations de démolition sont à l'arrêt », Al Huffington Post MaghrebMaroc, 4 octobre 2018.

${ }^{72}$ Des processus de politisation similaires sont mis en évidence dans : L. Zaki, Pratiques politiques au bidonville, Casablanca (2000-2005), thèse de doctorat en science politique, Paris, Institut d'études politiques, 2005.
} 
du droit». Il n'est pas question de détruire les biens publics ou de nuire à la « chère patrie ». Dès lors, la seule option consiste à quitter un pays où les «droits" des citoyens ne sont pas respectés. Il existe aussi plusieurs traces d'appropriation du savoir protestataire que le Mouvement du 20 février a contribué à cristalliser en $2011^{73}$, et dont la diffusion s'amplifie à travers les réseaux sociaux: la présence de haut-parleurs qui permettent d'unifier l'action protestataire; la distribution de pancartes blanches, plastifiées, en format standardisé A3, imprimées en rouge, mais sans logo ; les gilets orange revêtus par des hommes qui assurent le service d'ordre. De même, le vocabulaire employé et les slogans scandés attestent de la routinisation de l'héritage des mouvements de gauche. Les prises de paroles sont ponctuées par le mot «militant» (munadil) et par le «salut militant» (tahiyya nidaliyya). Il est question de « lutte militante continuelle» (an-nidal al-mustamirr), de « marcher de l'avant» (ila al-amam), « jusqu'au triomphe », et l'expression «nous résistons » (samidun) est peinte en rouge sur les murs. Des chants protestataires sont complétés par des strophes en adéquation avec la situation du douar. Pour finir, la foule scande le slogan phare du Hirak du Rif ${ }^{74}$ : « la mort plutôt que l'humiliation» (al-mawt wala al-madalla). Un porte-parole du douar dénonce «la tyrannie », fait appel aux «masses populaires », et alerte le « peuple marocain » :

« Je dis au peuple marocain, je le dis et je le répète : vous avez été dévoré le jour où le taureau blanc a été dévoré. Je leur rappelle un point : vous avez laissé tomber le Rif et ses enfants ont été exposés à la prison et à l'exil. Aujourd'hui, ce sont les habitants de Casablanca qui font appel à vous. [...] Rejoignez les enfants du peuple (wlad acha 'b) [...]. L'histoire demandera des comptes aux lâches. [...] Non à la hogra, non à la hogra ${ }^{75}$.»

Parallèlement à ce registre, des habitants puisent également dans le répertoire religieux pour dénoncer l'injustice. Des femmes répètent en chœur une citation coranique, habituellement déclamée pour solliciter un bienfait ou réprouver une nuisance, et souvent scandée pendant les manifestations encadrées par des organisations à référentiel islamique pour dénoncer l'injustice ou la répression : «Dieu nous suffit, il est notre meilleur garant » (hasbuna allah wa ni 'ma alwakil) ${ }^{76}$.

En somme, les paroles et les actions filmées dans cette vidéo trahissent des processus qui vont au-delà d'une "politisation de la contrainte ${ }^{77}$ ». Si la désingularisation et la conflictualisation sont repérables (identification d'une injustice, d'un «nous », d'un « eux », la désignation de responsables et de solutions), les habitants du douar manifestent également leur capacité à décrypter et à détourner les discours politiques officiels. De même, témoignent-ils d'une forte acculturation au registre du droit et au répertoire protestataire dont la diffusion s'est amplifiée à travers les réseaux sociaux. À cet égard, un militant de la section locale de l'Association marocaine des droits humains confirme que les habitants de ces douars ont organisé leurs

\footnotetext{
${ }^{73}$ M. Bennani-Chraïbi et M. Jeghllaly, «La dynamique protestataire du Mouvement du 20 février à Casablanca », Revue française de science politique, $\mathrm{n}^{\circ}$ 5-6, vol. 62, 2012, p. 867-894.

${ }^{74}$ Le 28 octobre 2016, Mouhcine Fikri, un vendeur de poisson âgé de 31 ans, est mort broyé dans une benne à ordures à Al Hoceïma, en essayant de sauver de la destruction sa marchandise saisie par les autorités portuaires. À la suite de ce drame, le nord du Maroc connaît 700 événements protestataires au cours des sept premiers mois du mouvement. Le bilan est lourd : deux morts, des dizaines de blessés graves, des centaines d'arrestations, de lourdes peines de prison, dont 20 ans pour les figures du mouvement. Voir par exemple M. Masbah, «A New Generation of Protests in Morocco? How Hirak al-Rif Endures », Policy Alternatives, Arab Reform Initiative, $2017<$ https://archives.arab-reform.net/en/node/1102>.

${ }^{75}$ Cette notion renvoie à la fois au mépris, à l'humiliation, à l'injustice et à l'oppression. Elle fait partie du langage politique des dominés aussi bien en Algérie qu'au Maroc. Sur l'analyse de cet événement, voir R. Zaireg, 2018, «Maroc : Face à la faillite politique, ne reste que la protestation », Yabiladi.com, 13 octobre 2018.

${ }^{76}$ Formulation qui se retrouve partiellement dans plusieurs sourates du Coran; voir par exemple : « Al Imran », verset 173.

${ }^{77}$ F. Vairel et L. Zaki, « Politisation sous contrainte et politisation de la contrainte : outsiders politiques et outsiders de la ville au Maroc», Critique internationale, $\mathrm{n}^{\circ}$ 50, vol. 1, 2011, p. 91-108.
} 
protestations de manière autonome ${ }^{78}$. Selon lui, bien que ces zones aient pendant longtemps constitué des réservoirs électoraux pour « les partis du Makhzen » (à savoir proche du Palais et de ses extensions), l'imminence du délogement et l'inefficience des relations clientélaires auraient incité leurs habitants à changer de stratégie, voire à reconnaître au Hirak du Rif une légitimité qu'ils ne lui auraient pas toujours concédée. Comme dans d'autres cas observés à la même époque, le rapport à la patrie est au cœur de la protestation, et l'appartenance nationale est conditionnée par l'exercice d'une citoyenneté pleine.

En définitive, l'analyse de cet épisode permet de souligner plusieurs points. En premier lieu, il importe de ne pas naturaliser un registre du politique en particulier et de garder en tête que les acteurs peuvent recourir - simultanément ou successivement - à plusieurs registres, qu'il s'agisse de s'opposer à l'ordre établi ou de le conforter. Ce faisant, il devient possible de dépister les réappropriations de même que les circulations des acteurs, des pratiques, des technologies et des cadrages dans une perspective généalogique que les filiations soient exhibées, redécouvertes, réinventées a posteriori par les acteurs, ou qu'elles soient occultées et invisibilisées. Deuxièmement, cet exemple démontre une nouvelle fois que les relations de clientèle sont instables et que les mêmes acteurs peuvent passer de la loyauté à la (menace de) défection ou à la protestation. Troisièmement, dans une perspective diachronique, cette action attire l'attention sur les apprentissages et les ressources que les dominés peuvent accumuler, même si cela est loin de se traduire systématiquement par la transformation des rapports de domination.

\section{CONCLUSION}

Depuis le milieu des années 1990, les appels d'air(e) se sont indubitablement multipliés et les études africaines ont contribué à féconder les débats. Au terme de cet article, nous espérons avoir montré que s'il est fondamental de faire voyager les questionnements, il est tout aussi important de se garder de supprimer un « savoir [spécifique] au service de fausses analogies ${ }^{79}$ ». Aussi, la première condition d'un dialogue fructueux autour de la notion de politisation est d'éviter de naturaliser, d'universaliser ou d'ériger en étalon une histoire singulière du politique. Il importe plutôt d'appréhender ces phénomènes en prêtant attention à «l'historicité vernaculaire du politique ${ }^{80} \gg$, aux trajectoires du politique qui se dessinent et se redessinent au croisement de «dynamiques du dedans » et « du dehors », tout en se donnant les moyens de saisir l'agentivité des acteurs. À partir de là, il devient vain d'opposer «la politique» et «l'infrapolitique », ou encore les perspectives de la politisation restrictives et extensives. Pour notre part, nous plaidons pour une analyse synchronique et diachronique des politisations différentielles des individus, des collectifs et des pratiques, qui soit attentive aux tâtonnements, aux intrications, aux circulations et aux hybridations entre des registres d'(in)action pluriels, qui se déploient dans différents sites d'interactions ${ }^{81}$.

\footnotetext{
${ }^{78}$ Échange téléphonique avec l'autrice, en octobre 2018.

${ }^{79}$ Ch. Tilly, «Forward», dans Q. Wiktorowicz (ed.), Islamic Activism. A Social Movement Theory Approach, Bloomington, Indiana University Press, 2004, p. IX-XII.

${ }^{80}$ R. Bertrand, «Politiques du moment colonial. Historicités indigènes et rapports vernaculaires au politique en "situation coloniale" », Questions de recherche/Research in question, n 26, octobre 2008.

${ }^{81}$ Je tiens à remercier pour leur lecture attentive d'une version initiale de ce texte Myriam Catusse et Bernard Voutat.
} 